\title{
A UHPLC-MS/MS Method for the Quantitation of Olmutinib in Rat Plasma
}

\author{
Su-su Bao, Jian Wen, Teng-hui Liu, Bo-wen Zhang, Chen-chen Wang and Guo-xin Hu* \\ School of Pharmaceutical Science, Wenzhou Medical University, Wenzhou, Zhejiang, China
}

Received: 13 September 2017; accepted: 10 December 2017

\begin{abstract}
Olmutinib $\left(\right.$ Olita $\left.^{\mathrm{TM}}\right)$ is an oral third-generation epidermal growth factor receptor tyrosine kinase inhibitor (EGFR-TKI) which is used to treat non-small cell lung cancer (NSCLC). A simple, rapid, and sensitive method based on ultraperformance liquid chromatography tandem mass spectrometry (UHPLC-MS/MS) has been developed for the determination of olmutinib. Sample preparation was performed following simple one-step protein precipitation with acetonitrile. Olmutinib and internal standard (dasatinib) were separated on an Eclipse Plus C18 RRHD $(2.1 \times 50 \mathrm{~mm}, 1.8 \mu \mathrm{m})$ column. The mobile phase consisted of acetonitrile $-0.1 \%$ formic acid in water with gradient elution. A total run time of $1.7 \mathrm{~min}$ was achieved. Detection was performed on a positive-ion electrospray ionization mass spectrometer in multiple reaction monitoring (MRM) mode, using transitions of $\mathrm{m} / \mathrm{z} 487.2 \rightarrow 402.1$ for olmutinib and $\mathrm{m} / \mathrm{z} 488.2 \rightarrow 401$ for dasatinib (IS), respectively. The calibration curve $\left(R^{2}=0.999\right)$ was linear over the range of $1-500 \mathrm{ng} / \mathrm{mL}$. The recovery of olmutinib ranged from $85.8 \%$ to $95.5 \%$. This method can be applied to pharmacokinetic studies of olmutinib.
\end{abstract}

Keywords: Olmutinib, UHPLC-MS/MS, pharmacokinetics, non-small cell lung cancer

\section{Introduction}

Lung cancer is the leading cause of cancer death worldwide. Non-small cell lung cancer (NSCLC) is the most common type, accounting for more than $80 \%$ of all lung cancers $[1,2]$. NSCLC includes squamous cell carcinoma, adenocarcinoma, and large cell carcinoma. Compared with small cell lung cancer, NSCLC cells proliferate more slowly and metastasize later. Despite novel diagnostic and therapeutic advances, the 5-year survival rate for NSCLC remains approximately $15 \%$ [3].

Approximately $19 \%$ to $51.4 \%$ of NSCLC cases demonstrate overexpression of epidermal growth factor receptor (EGFR) [4, 5]. Some studies suggest that EGFR-activated cell signaling plays a significant role in lung tumorigenesis and tumor progression $[6,7]$. Therefore, inhibition of EGFR signaling has been suggested as an approach to NSCLC treatment [8-10]. Several EGFR tyrosine kinase inhibitors (EGFR-TKIs) have been developed. First-generation EGFR-TKIs such as gefitinib and erlotinib give good primary responses against activating EGFR mutations by binding to the tyrosine kinase (TK) domain of EGFR. Unfortunately, many patients acquire resistance during the first year of treatment [11, 12]. Second-generation EGFR-TKIs such as afatinib and dacomitinib bind irreversibly to the TK domain of the EGFR and other receptors of the ERbB family $[12,13]$. These newer agents also do not prevent development of tumor resistance. This leads to the development of third-generation TKIs that target the EGFR T790M mutation, an important resistance mechanism against EGFR-TKIs [12, 13].

Olmutinib (Olita ${ }^{\mathrm{TM}}$ ) is an oral third-generation EGFR mutantselective TKI developed by Hanmi Pharmaceutical Co. Ltd. It was approved in South Korea on May 13, 2016 (Figure 1) [14]. Oral olmutinib is absorbed well. In EGFR mutation-positive NSCLC patients, median time to maximum serum concentration is $2.5-5.9 \mathrm{~h}$. Mean half-life is $8.1-18.3 \mathrm{~h}$ with a dosage range of 75 to $1200 \mathrm{mg}$ /day. In phase I/II trials, adverse events (AEs) caused by olmutinib included diarrhea and prur-

* Author for correspondence: Wenzhou Medical University, Chashan University Town, Ouhai District, China; hgx@wmu.edu.cn itis, but there were no reports of hyperglycemia, QT prolongation, or treatment-related deaths [14].

Based on the above background, olmutinib will be a hot spot in the field of NSCLC in the future and, as a new drug, more and more experiments about olmutinib will be done. However, a method for determination and pharmacokinetic investigation of olmutinib has not yet been reported. In this article, we describe a quantitative analysis of olmutinib using UHPLC-MS/MS.

\section{Experimental}

Compounds. Olmutinib ( $>99 \%$ pure) was obtained from Perfemiker. The internal standard (IS) dasatinib was acquired from Toronto Research Chemicals (TRC). Methanol (liquid chromatography-mass spectrometry [LC-MS] grade) and acetonitrile (LC-MS grade) were supplied by Merck Company (Darmstadt, Germany). About $0.1 \%$ formic acid (99.9\% purity) was obtained from J \& K Scientific Ltd. (Shanghai, China). Ultrapure water was prepared by a Milli-Q system (Millipore, Bedford, MA).

Chromatography and MS/MS Method. Chromatographic analysis was performed on an Agilent 1290 system (Agilent Technologies, USA). Partial loop injections of $2 \mu \mathrm{L}$ were made on an Eclipse Plus C18 RRHD $(2.1 \times 50 \mathrm{~mm}, 1.8 \mu \mathrm{m})$ column. The compounds were eluted by $0.1 \%$ formic acid in water (A) and acetonitrile (B). The $1.7 \mathrm{~min}$ gradient was run as follows: $27-95 \%$ B (0-0.6 min), maintained at 95\% B (0.6-1.6 min), and 95-27\% B (1.6-1.7 $\mathrm{min})$. The total run time $(3.3 \mathrm{~min})$ included stop time $(1.7 \mathrm{~min})$ and post time $(1.6 \mathrm{~min})$. The flow rate was $0.4 \mathrm{~mL} / \mathrm{min}$.

The sample was tested on an Agilent G6420A Triple quadrupole LC-MS (manufactured in Singapore using domestic and foreign components) equipped with an electrospray ionization (ESI) source. The fragmentor voltage was $180 \mathrm{~V}$ for olmutinib and $214 \mathrm{~V}$ for dasatinib. Olmutinib was monitored at $\mathrm{m} / \mathrm{z} 487.2 \rightarrow$ 402.1 at $38 \mathrm{~V}$ collision energy, and dasatinib (IS), at $\mathrm{m} / \mathrm{z} 488.2 \rightarrow$ 401 at $33 \mathrm{~V}$ collision energy. The gas temperature was set to $350{ }^{\circ} \mathrm{C}$, and the flow rate was $10 \mathrm{~L} / \mathrm{min}$. The cell accelerator voltage for olmutinib was $3.0 \mathrm{kV}$. Dasatinib voltage was $5.0 \mathrm{kV}$.

This is an open-access article distributed under the terms of the Creative Commons Attribution-NonCommercial 4.0 International License (https://creativecommons.org/licenses/by-nc/4.0/), which permits unrestricted use, distribution, and reproduction in any medium for non-commercial purposes, provided the original author and source are credited, a link to the CC License is provided, and changes - if any - are indicated. 
<smiles>C=CC(=O)Nc1cccc(Oc2nc(Nc3ccc(N4CCN(C)CC4)cc3)nc3c2SCC3)c1</smiles>

A<smiles>Cc1nc(Nc2ncc(C(=O)Nc3c(C)cccc3Cl)s2)cc(N2CCN(CCO)CC2)n1</smiles>

B

Figure 1. The chemical structures of olmutinib and IS in the present study: (A) olmutinib and (B) dasatinib (IS)

Mass Hunter work station data acquisition software was used for data collection, and Qualitative Analysis software (version B.07.00) was used to process the data.

Preparation of Standard Solutions. A stock solution of olmutinib was prepared in methanol, which was then diluted with methanol to various concentrations as the working solution. Calibration standards and quality control (QC) samples were prepared by adding the above working solutions to blank rat plasma. Final calibration standards of olmutinib were prepared at $1,2,5,10,20,50,100,200$, and $500 \mathrm{ng} / \mathrm{mL}$. QC samples were set at $3 \mathrm{ng} / \mathrm{mL}, 40 \mathrm{ng} / \mathrm{mL}$, and $400 \mathrm{ng} / \mathrm{mL}$.

Sample Preparation. Ninety microliters of rat plasma and $10 \mu \mathrm{L}$ various concentrations working solutions of olmutinib were mixed in a $1.5-\mathrm{mL}$ tube. Then, $20 \mu \mathrm{L}$ dasatinib (IS, $10 \mu \mathrm{g} / \mathrm{mL}$ ) and $200 \mu \mathrm{L}$ acetonitrile were added. After mixing well and centrifuging, $150 \mu \mathrm{L}$ supernatant was collected and diluted by pure water for testing.

Pharmacokinetic Study. Six male Sprague-Dawley rats (240-310 g) were provided by the Laboratory Animal Center of Wenzhou Medical University (Wen-Zhou, China) to perform the pharmacokinetic study of olmutinib. All rats were fasted overnight. Olmutinib was dissolved in dimethyl sulfoxide (DMSO) (modicum, since DMSO is toxic) and edible oil. Following an oral dose of $50 \mathrm{mg} / \mathrm{kg}, 300 \mu \mathrm{L}$ of blood samples was drawn from rat tail vein. Samples were obtained at $10 \mathrm{~min}$, 30 min, 1 h, 2 h, 4 h, 6 h, 8 h, 10 h, 12 h, 24 h, and 36 h.

Method Validation. Validation of the method was carried out according to a laboratory scheme published by the Food and Drug Administration (FDA) and European Medicines Agency (EMA).

A nine-point calibration curve $(1-500 \mathrm{ng} / \mathrm{mL})$ was analyzed by means of the least squares linear regression $(1 / \times 2$ weighted), using the peak area ratio against concentrations. Coefficient $\left(r^{2}\right)$ values was 0.999 , which was better than 0.985 . The lower limit of quantification (LLOQ) was determined as the lowest concentration on the calibration curves.

Intra-day and inter-day accuracy and precision were determined by analysis of six replicates of QC samples over 3 days. Relative standard deviation (RSD \%) was calculated for precision, while relative error (RE \%) was determined for accuracy. Both RSD $\%$ and RE $\%$ are expected to be less than $\pm 15 \%$.

Selectivity of this method was analyzed by comparing the results of six batches of blank plasma samples, rat plasma samples, and blank sample containing olmutinib and dasatinib (IS). The purpose of the analysis was to test for the presence of endogenous interference introduced by blank plasma.

To evaluate the recovery of olmutinib, two types of peak areas were examined. One was from six QC samples to which olmutinib was added prior to the precipitation reaction of blank plasma. The second was obtained from samples to which olmutinib was added following protein precipitation. The matrix effect was analyzed according to the ratio of the peak areas of the analytes containing the same amount of blank rat plasma dissolved in standard solution.
The stability of olmutinib in plasma samples was determined under different storage conditions as follows: maintained room temperature for $4 \mathrm{~h}$ and at $4{ }^{\circ} \mathrm{C}$ for $24 \mathrm{~h}$, cryopreserved at $-20{ }^{\circ} \mathrm{C}$ for 15 days, and processed through three freeze-thaw cycles (thawing at room temperature and refreezing at $-20{ }^{\circ} \mathrm{C}$ ).

\section{Results and Discussion}

Method Development. A reliable mass spectrometry method was established based on various previous trials. MS responses for olmutinib and dasatinib (IS) were better in positive ionization mode versus negative ionization mode. To optimize the mass spectrometry method, collision energy (CE) was adjusted from 5 to 45 , and fragmentor voltage was changed from $50 \mathrm{~V}$ to $220 \mathrm{~V}$. As a result, olmutinib was monitored at $\mathrm{m} / \mathrm{z} 487.2 \rightarrow$ 402.1 at $38 \mathrm{~V}$ collision energy, and dasatinib (IS) was at $\mathrm{m} / \mathrm{z}$ $488.2 \rightarrow 401$ at $33 \mathrm{~V}$ collision energy. More details of spectrometric parameters are displayed in Table 1 and Figure 2.

Chromatographic conditions included constitution of mobile phase and column. Liquid phase schemes included water,

Table 1. MS parameters for olmutinib and dasatinib

\begin{tabular}{lcccc}
\hline $\begin{array}{l}\text { Compound } \\
\text { name }\end{array}$ & $\begin{array}{c}\text { Precursor } \\
\text { ion }(\mathrm{m} / \mathrm{z})\end{array}$ & $\begin{array}{c}\text { Product } \\
\text { ion }(\mathrm{m} / \mathrm{z})\end{array}$ & $\begin{array}{c}\text { Collision } \\
\text { energy }\end{array}$ & Fragmentor \\
\hline Olmutinib & 487.2 & 402.1 & 38 & 180 \\
Dasatinib & 488.2 & 401 & 33 & 214 \\
\hline
\end{tabular}

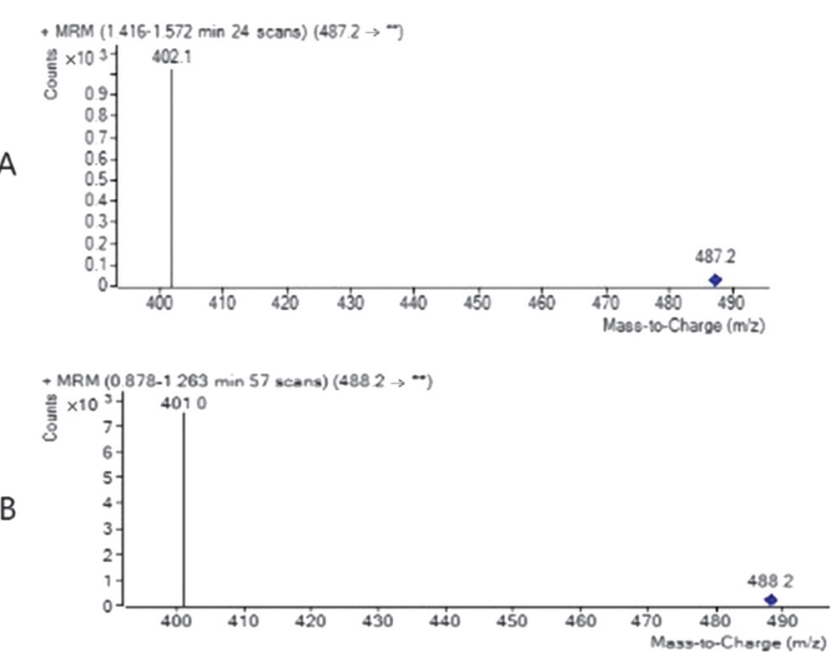

Figure 2. Product ion mass spectra of olmutinib (A) and dasatinib (IS) (B)

Table 2. Precision and accuracy for olmutinib of QC sample in rat plasma $(n=6)$

\begin{tabular}{ccccccc}
\hline Analytes & \multirow{2}{L}{$\begin{array}{c}\text { Level } \\
(\mathrm{ng} / \mathrm{mL})\end{array}$} & \multicolumn{2}{c}{ Intra-day precision } & & \multicolumn{2}{c}{ Inter-day precision } \\
\cline { 3 - 4 } \cline { 6 - 7 } & & $\mathrm{RE}(\%)$ & $\mathrm{RSD}(\%)$ & & $\mathrm{RE} \mathrm{( \% )}$ & $\mathrm{RSD}(\%)$ \\
\hline Olmutinib & $3 \mathrm{ng} / \mathrm{mL}$ & 2.0 & 10.0 & & -1.1 & 2.2 \\
& $40 \mathrm{ng} / \mathrm{mL}$ & -9.8 & 1.8 & & -11.9 & 5.8 \\
& $400 \mathrm{ng} / \mathrm{mL}$ & 12.0 & 3.3 & & 10.5 & 1.4 \\
\hline
\end{tabular}



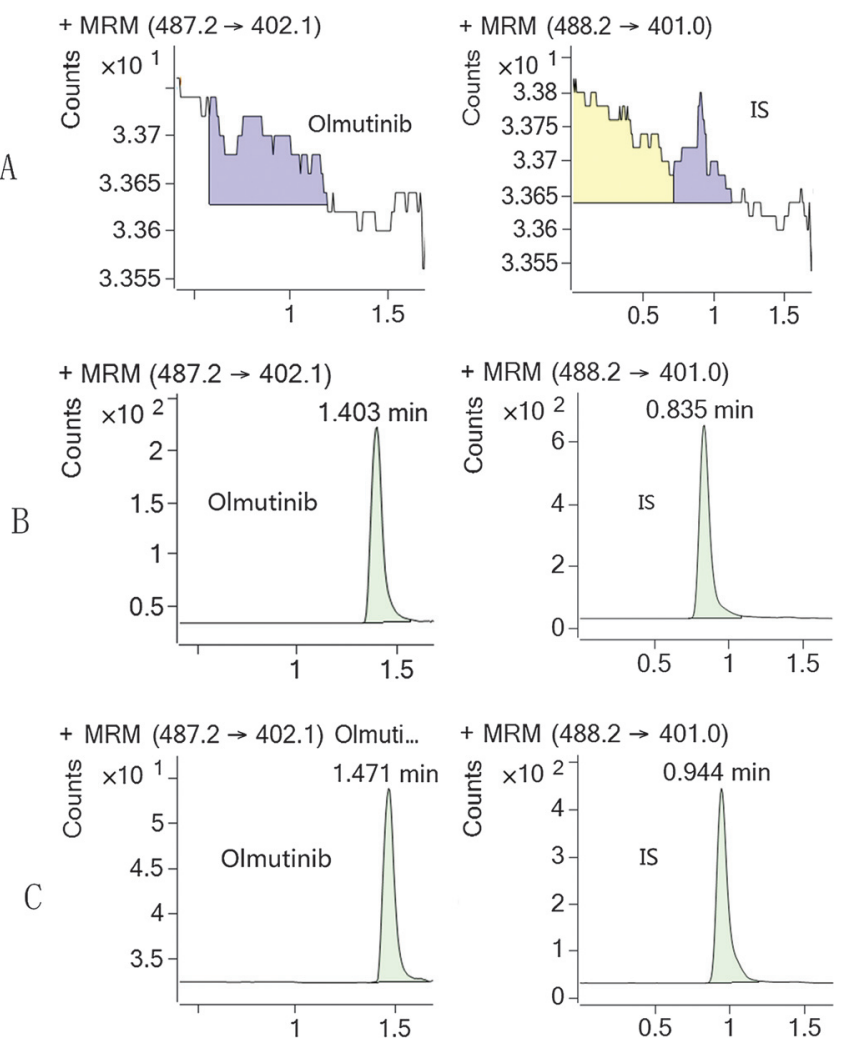

Figure 3. Representative chromatograms of olmutinib and IS in rat plasma sample. (A) A blank plasma sample; (B) a blank plasma sample spiked with olmutinib and IS; and (C) a rat plasma sample obtained $1 \mathrm{~h}$ after oral administration of olmutinib

Table 3. Recovery and matrix effect for olmutinib of QC sample in rat plasma $(n=6)$

\begin{tabular}{lccc}
\hline Analytes & Level (\%) & Recovery $(\%)$ & Matrix effect (\%) \\
\hline Olmutinib & $3 \mathrm{ng} / \mathrm{mL}$ & 85.8 & 102.3 \\
& $40 \mathrm{ng} / \mathrm{mL}$ & 95.8 & 96.9 \\
& $400 \mathrm{ng} / \mathrm{mL}$ & 95.5 & 96.9 \\
\hline
\end{tabular}

acetonitrile, and water with $0.1 \%$ formic acid. The result was that acetonitrile and water with $0.1 \%$ formic gave good peak shape without distinct peak broadening or tailing. Gradient elution, as opposed to isocratic elution, was used to obtain shorter retention times. Flow rate of liquid phase was tried between 0.2 and $0.4 \mathrm{~mL} / \mathrm{min}$. Column temperature changed from 25 to $45^{\circ} \mathrm{C}$.

Method Validation. The calibration curve of olmutinib $(n=9)$ was obtained from the concentration range of $1-500 \mathrm{ng} / \mathrm{mL}$, with a coefficient of determination $R^{2}=0.999$, demonstrating good linearity. The LLOQ was determined as the lowest concentration on the calibration curves.

Intra-day and inter-day RSD (representing precision) as well as intra-day and inter-day RE (representing accuracy) were within the $\pm 15 \%$ range as required. The extraction recoveries were good, and the matrix effect could be ignored. All the details were showed in Table 2.

To evaluate selectivity of the assay, blank rat plasma samples, blank rat plasma mixed with olmutinib and dasatinib (IS), and a rat plasma sample were analyzed. The chromatograms obtained from these samples are shown in Figure 3. No obvious interferences were found at the retention time of olmutinib $(1.4 \mathrm{~min})$ and internal standard $(0.8 \mathrm{~min})$.

The values of the extraction recoveries and the matrix effects of olmutinib were showed in Table 3. The stability of olmutinib was tested under different conditions. QC samples were kept at room temperature for $4 \mathrm{~h}$, at $4{ }^{\circ} \mathrm{C}$ for $24 \mathrm{~h}$, and processed in three freeze-thaw cycles to examine short-term stability. Cryopreservation at $-20{ }^{\circ} \mathrm{C}$ for 15 days was examined to test long-term stability. The results were displayed in Table 4, demonstrating that no obvious change of olmutinib appeared in rat plasma in all storage conditions.

Method Application. The developed UHPLC-MS/MS method was successfully applied to the plasma sample analysis in a pharmacokinetic study of olmutinib. Six SD rats were employed to obtain a concentration-time curve (Figure 4) following oral administration of olmutinib (50 $\mathrm{mg} / \mathrm{kg})$. Pharmacokinetic parameters were analyzed by DAS software (version 3.0) using non-compartmental mode. Complete data are displayed in Table 5.

\section{Conclusions}

A UHPLC-MS/MS method with good selectivity and sensitivity has been successfully established and validated. This method is simple, rapid, and sensitive, with good linearity,

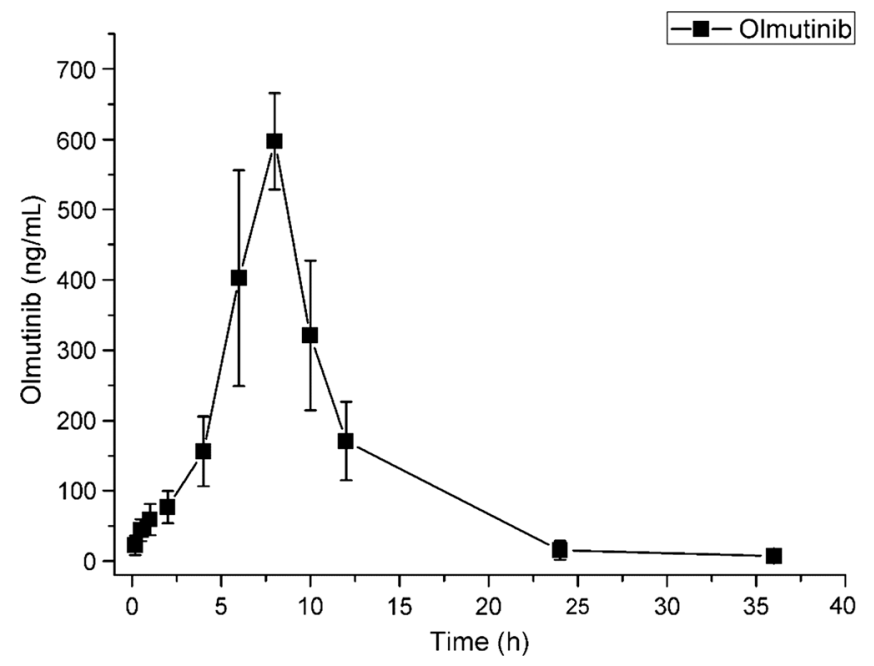

Figure 4. Mean plasma concentration-time curves after oral administration $(50 \mathrm{mg} / \mathrm{kg})$ of olmutinib in rats

Table 5. The pharmacokinetic parameters of olmutinib in rat plasma after oral administration

\begin{tabular}{lccc}
\hline Parameters & Unit & \multicolumn{2}{c}{ Mean } \\
\cline { 3 - 4 } & & \multicolumn{2}{c}{ po $50 \mathrm{mg} / \mathrm{kg}$} \\
\hline AUC $(0-t)$ & $\mu \mathrm{g} / \mathrm{L} * \mathrm{~h}$ & 4562.5 & 975.5 \\
AUC $(0-\infty)$ & $\mu \mathrm{g} / \mathrm{L} * \mathrm{~h}$ & 4584.2 & 971.0 \\
$t_{1 / 2}$ & $\mathrm{~h}$ & 3.6 & 1.4 \\
$T_{\max }$ & $\mathrm{h}$ & 7.7 & 0.82 \\
$V$ & $\mathrm{~L} / \mathrm{kg}$ & 60.3 & 32.5 \\
$\mathrm{CL}$ & $\mathrm{L} / \mathrm{h} / \mathrm{kg}$ & 11.9 & 2.4 \\
$C_{\max }$ & $\mu \mathrm{g} / \mathrm{L}$ & 597.4 & 68.5 \\
\hline
\end{tabular}

Table 4. Summary of stability of olmutinib in rat plasma under different storage conditions $(n=6)$

\begin{tabular}{|c|c|c|c|c|c|c|c|c|c|}
\hline \multirow[t]{2}{*}{ Analytes } & \multirow[t]{2}{*}{ Level (\%) } & \multicolumn{2}{|c|}{ Room temperature } & \multicolumn{2}{|c|}{$4{ }^{\circ} \mathrm{C}$} & \multicolumn{2}{|c|}{ Three freeze-thaw } & \multicolumn{2}{|c|}{$-20^{\circ} \mathrm{C}$} \\
\hline & & RE (\%) & RSD (\%) & RE (\%) & RSD (\%) & RE (\%) & RSD (\%) & RE (\%) & RSD (\%) \\
\hline Olmutinib & $\begin{array}{c}3 \mathrm{ng} / \mathrm{mL} \\
40 \mathrm{ng} / \mathrm{mL} \\
400 \mathrm{ng} / \mathrm{mL}\end{array}$ & $\begin{array}{r}6.3 \\
-13.4 \\
11.8\end{array}$ & $\begin{array}{r}11.0 \\
7.8 \\
3.0\end{array}$ & $\begin{array}{r}5.4 \\
-8.3 \\
8.9\end{array}$ & $\begin{array}{r}12.9 \\
2.1 \\
1.6\end{array}$ & $\begin{array}{r}7.5 \\
-4.5 \\
1.0\end{array}$ & $\begin{array}{l}4.5 \\
4.5 \\
2.6\end{array}$ & $\begin{array}{l}7.3 \\
5.0 \\
8.9\end{array}$ & $\begin{array}{r}10.8 \\
5.9 \\
1.6\end{array}$ \\
\hline
\end{tabular}


precision, accuracy, recovery, and stability. The method was successfully employed in a pharmacokinetic study in rats. Furthermore, this method can be used as a reference for the therapeutic drug monitoring. To our knowledge, this is the first report of a validated assay for olmutinib.

\section{References}

1. Torre, L. A.; Bray, F.; Siegel, R. L.; Ferlay, J.; Lortet-Tieulent, J.; Jemal, A. Ca-Cancer J. Clin. 2015, 65, 87-108.

2. Chen, W. Q.; Zhang, S. W.; Zou, X. N.; Zhao, P. Chin. J. Cancer Res. 2011, 23, 3-9.

3. D'Arcangelo, M.; Hirsch, F. R. Biol. Targets Ther. 2014, 8, 183-192.

4. Weiss, J. M.; Villaruz, L. C.; Socinski, M. A.; Ivanova, A.; Grilley-Olson, J.; Dhruva, N.; Stinchcombe, T. E. Lung Cancer 2014, 86, 288-290.
5. Shi, Y.; Au, J. S.; Thongprasert, S.; Srinivasan, S.; Tsai, C. M.; Khoa, M. T.; Heeroma, K.; Itoh, Y.; Cornelio, G.; Yang, P. C. J. Thorac. Oncol. 2014, 9, $154-162$.

6. Filosto, S.; Becker, C. R.; Goldkorn, T. Mol. Cancer Ther. 2012, 11, 795-804.

7. Chou, Y. T.; Lin, H. H.; Lien, Y. C.; Wang, Y. H.; Hong, C. F.; Kao, Y. R.; Lin, S. C.; Chang, Y. C.; Lin, S. Y.; Chen, S. J.; Chen, H. C.; Yeh, S. D.; Wu, C. W. Cancer Res. 2010, 70, 8822-8831.

8. Laskin, J. J.; Sandler, A. B. Cancer Treat. Rev. 2004, 30, 1-17.

9. Glover, K. Y.; Perez-Soler, R.; Papadimitradopoulou, V. A. Semin. Oncol. 2004, 31, 83-92.

10. Haber, D. A.; Bell, D. W.; Sordella, R.; Kwak, E. L.; Godin-Heymann, N.; Sharma, S. V.; Lynch, T. J.; Settleman, J. Cold Spring Harbor Symp. Quant. Biol. 2005, 70, 419-426.

11. Peters, S.; Zimmermann, S.; Adjei, A. A. Cancer Treat. Rev. 2014, 40, 917-926.

12. Tan, C. S.; Gilligan, D.; Pacey, S. Lancet Oncol. 2015, 16, e447-e459.

13. Liao, B. C.; Lin, C. C.; Yang, J. C. Curr. Opin. Oncol. 2015, 27, 94-101.

14. Kim, E. S. Drugs 2016, 76, 1153-1157. 